Article

\title{
Synthesis, Spectroscopic Characterization, and In Vitro Antibacterial Evaluation of Novel Functionalized Sulfamidocarbonyloxyphosphonates
}

\author{
Abdeslem Bouzina ${ }^{1}$, Khaoula Bechlem ${ }^{1}$, Hajira Berredjem ${ }^{2}$, Billel Belhani ${ }^{1}$, Imène Becheker ${ }^{2}$, \\ Jacques Lebreton ${ }^{3}$, Marc Le Borgne ${ }^{4}$ (D) , Zouhair Bouaziz ${ }^{4}$, Christelle Marminon ${ }^{4 * *}$ (iD \\ and Malika Berredjem ${ }^{1, *}$
}

1 Laboratory of Applied Organic Chemistry, Synthesis of Biomolecules and Molecular Modelling Group, Badji-Mokhtar-Annaba University, Box 12, 23000 Annaba, Algeria; bouzinaabdeslem@yahoo.fr (A.B.); bechlemkhaoula92@yahoo.com (K.B.); billelbelhani@yahoo.com (B.B.)

2 Laboratory of Applied Biochemistry and Microbiology, Department of Biochemistry, Badji-Mokhtar-Annaba University, Box 12, 23000 Annaba, Algeria; hajira.berredjem@univ-annaba.dz (H.B.);

i_mene7@msn.com (I.B.)

3 CNRS, Université de Nantes, Chimie et Interdisciplinarité: Synthèse, Analyse, Modélisation (CEISAM), UMR CNRS 6230, 2 rue de la Houssinière, BP92208, CEDEX 3, 44322 Nantes, France; jacques.lebreton@univ-nantes.fr

4 Université de Lyon, Université Lyon 1, Faculté de Pharmacie-ISPB, EA 4446 Bioactive Molecules and Medicinal Chemistry, SFR Santé Lyon-Est CNRS UMS3453-INSERM US7, CEDEX 8, 69373 Lyon, France; marc.le-borgne@univ-lyon1.fr (M.L.B.); zouhair.bouaziz@univ-lyon1.fr (Z.B.)

* Correspondence: christelle.marminon-davoust@univ-lyon1.fr (C.M.); malika.berredjem@univ-annaba.org (M.B.); Tel.: +33-478772867 (C.M.); +213-38876567 (M.B.)

Received: 18 June 2018; Accepted: 5 July 2018; Published: 10 July 2018

\begin{abstract}
Several new sulfamidocarbonyloxyphosphonates were prepared in two steps, namely carbamoylation and sulfamoylation, by using chlorosulfonyl isocyanate (CSI), $\alpha$-hydroxyphosphonates, and various amino derivatives and related (primary or secondary amines, $\beta$-amino esters, and oxazolidin-2-ones). All structures were confirmed by ${ }^{1} \mathrm{H},{ }^{13} \mathrm{C}$, and ${ }^{31} \mathrm{P} \mathrm{NMR}$ spectroscopy, IR spectroscopy, and mass spectroscopy, as well as elemental analysis. Eight compounds were evaluated for their in vitro antibacterial activity against four reference bacteria including Gram-positive Staphylococcus aureus (ATCC 25923), and Gram-negative Escherichia coli (ATCC 25922), Klebsiella pneumonia (ATCC 700603), Pseudomonas aeruginosa (ATCC 27853), in addition to three clinical strains of each studied bacterial species. Compounds $\mathbf{1 a}-\mathbf{7} \mathbf{a}$ and $\mathbf{1} \mathbf{b}$ showed significant antibacterial activity compared to sulfamethoxazole/trimethoprim, the reference drug used in this study.
\end{abstract}

Keywords: sulfamides; phosphonates; carbamoylation; sulfamoylation; antibacterial activity

\section{Introduction}

The synthesis and reactivity of sulfamides (sulfonyl analogues of ureas) have attracted much interest in the last decades [1]. A large number of sulfamide derivatives have been reported to show biological activities such as anti-mycobacterial, anticonvulsant, anti-hypoglycemic, anticancer, and enzyme inhibition (e.g., carbonic anhydrase I, HIV-1 protease, elastase, carboxypeptidase A) [2-9]. These important compounds have been synthesized by various routes, most of them using the reaction of a sulfonyl chloride with ammonia or primary and secondary amines [10]. Another approach utilizes the amide exchange of a sulfamide by heating with an amine [11]. In parallel, many synthetic efforts have also focused on sulfonamide derivatives that have shown great potency to inhibit important 
biological targets such as cox-2, carbonic anhydrase (e.g., isoenzymes I, II, VII, IX), and NaV1.7, or to block, for example, the Chlamydia fatty acid synthesis [12-16].

In addition, the extensive interest in the synthesis of bifunctional sulfonamide or sulfamide-phosphonate derivatives is due to their broad biological activities. In Figure 1, the structures of six bifunctional compounds are depicted. Biasone et al. [17] demonstrated that analogues of $\alpha$-biphenylsulfonylamino 2-methylpropyl phosphonate $\mathbf{1}$ exhibit potency against several matrix metalloproteinases (MMPs). New sildenafil analogue 2 containing a phosphonate group in the $5^{\prime}$-sulfonamide moiety of the phenyl ring has shown promising in vitro PDE5 inhibitory activity [18]. Sulfonamide derivative 3 containing a single difluoromethylene phosphonate group has been discovered to be a potent inhibitor of protein tyrosine phosphatase PTP1B [19]. A series of phosphonate derivatives of mycophenolic acid $\mathbf{4}$ were described as anticancer, antiviral, and anti-inflammatory agents [20]. Compound 5 shows the highest insecticidal activity against plant pests [21]. It should be pointed out that to the best of our knowledge, it is the only example of compounds containing a sulfamidocarbonyloxyphosphonate moiety described in the literature. Finally, Winum et al. [22] reported the synthesis of sulfamide analogues of fotemustine 6 along with preliminary in vitro evaluation on two human melanoma cell lines.

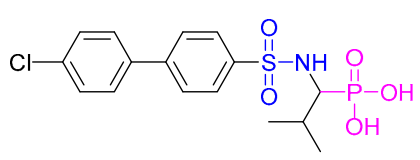

1

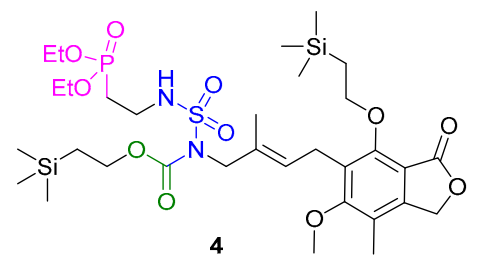

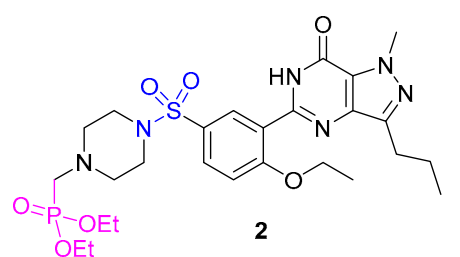
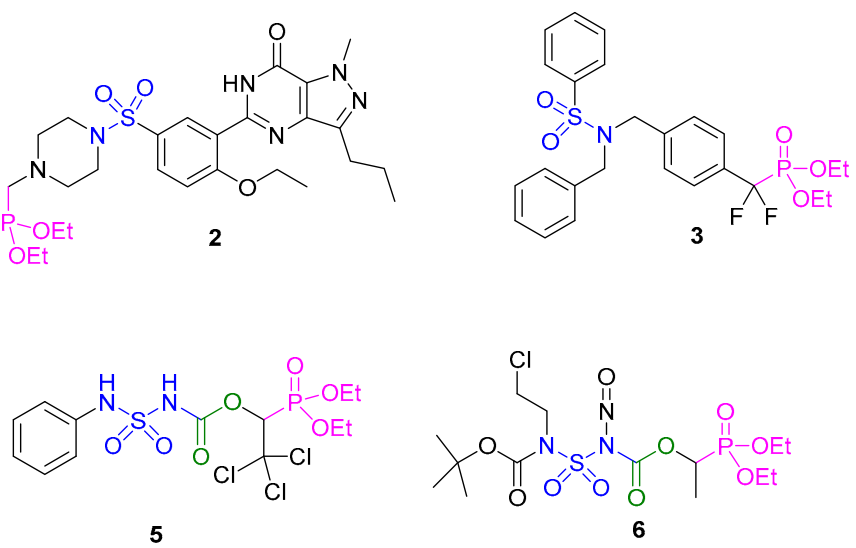

Figure 1. Structure of diverse sulfonamide and sulfamide derivatives containing a phosphonate-type group.

Since the 1930's, sulfamide and sulfonamide derivatives have had a special place in the anti-infectious strategies and their therapeutic application continues to be investigated, as illustrated by this recent work on the use of sulfonamide agents against Staphylococcus aureus (SA) of the CNS [23]. They demonstrated that sulfadiazine and sulfamethoxazole (SMX) (Figure 2) exhibited strong activity against bacteria. Fosfomycin (Figure 2) is another well-known antibacterial agent with a structure containing a phosphonate motif and may be prescribed alone or in combination (e.g., with vancomycin). Unfortunately, year after year, increased bacterial resistance to sulfonamides/sulfamides [24] to the combination sulfamethoxazole-trimethoprim (SMX-TMP) [25] and to fosfomycin [26] has limited their use. Moreover, the appearance of multidrug resistant Gram-positive bacteria, in particular methicillin-resistant Staphylococcus aureus (MRSA) and vancomycin-resistant Enterococci (VRE), has become a major health problem [27]. So, new research on drug discovery needs to be intensively developed for designing new antibacterial agents.
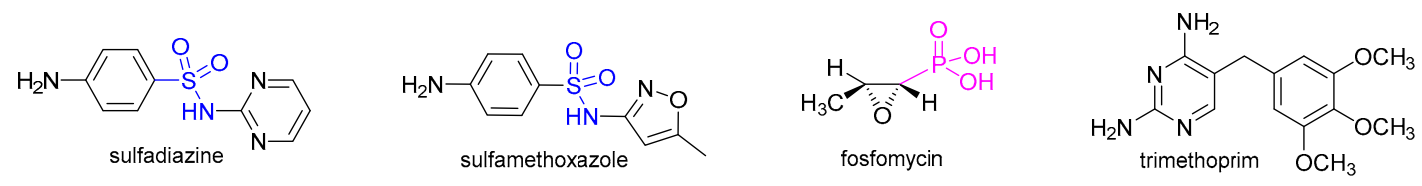

Figure 2. Structures of drugs approved for human use. 
In continuation of our interest in the preparation of sulfonamide and sulfamide derivatives [28-31], we decided to include both motifs, sulfamido and phosphonate, on each targeted compound and then to obtain new hybrids also containing an $\alpha$-phenyl on the phosphonate methylene. For this preliminary study, we opted to select a set of various substituents $-\mathrm{NR}_{2} \mathrm{R}_{3}$ and $-\mathrm{PO}(\mathrm{OR})_{2}$ in order to shape the first SAR trends in this series (Figure 3). To link these motifs, we chose a carbonyloxy-type spacer present in compounds 5 and 6 (Figure 1). The first-step reaction using chlorosulfonyl isocyanate and the corresponding $\alpha$-hydroxyphosphonate-type intermediate allowed us to synthesize all $N$-chlorosulfonyl carbamate intermediates. In the second step, the key structural sequence, sulfamidocarbonyloxyphosphonate, was achieved directly from various amines. The antibacterial activity of eight phosphonate derivatives (1a-7a and $\mathbf{1 b}$ ) was studied against representative reference strains Staphylococcus aureus ATCC 25923, Escherichia coli ATCC 25922, Klebsiella pneumoniae ATCC 700603, and Pseudomonas aeruginosa ATCC 27853, as well as diverse clinical strains. Inhibition zones were performed by the disc diffusion method and the MIC values were determined by the dilution broth method [32]. The combination SMX-TMP, currently employed to treat bacterial infections, was used as the reference standard.

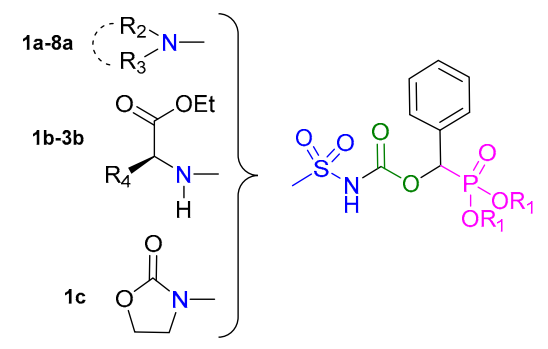

Figure 3. General formula of studied compounds.

\section{Results and Discussion}

\subsection{Chemistry}

The synthetic route for the preparation of a novel series of sulfamidocarbonyloxyphosphonates 1a-8a is outlined in Scheme 1. The synthesis was carried out in two steps. First, carbamoylation under anhydrous conditions of commercial chlorosulfonyl isocyanate with the corresponding $\alpha$-hydroxyphosphonate $\left(\mathrm{R}_{1}=\right.$ methyl or ethyl $)$, easily prepared in a single step [33,34], quantitatively afforded the corresponding $N$-chlorosulfonyl carbamate intermediate. Reaction with various primary or secondary amines in the presence of triethylamine at $0{ }^{\circ} \mathrm{C}$ then gave the target compounds $1 \mathbf{a}-8 \mathbf{a}$ in excellent yields (92-99\%) within 60-90 min (Table 1).

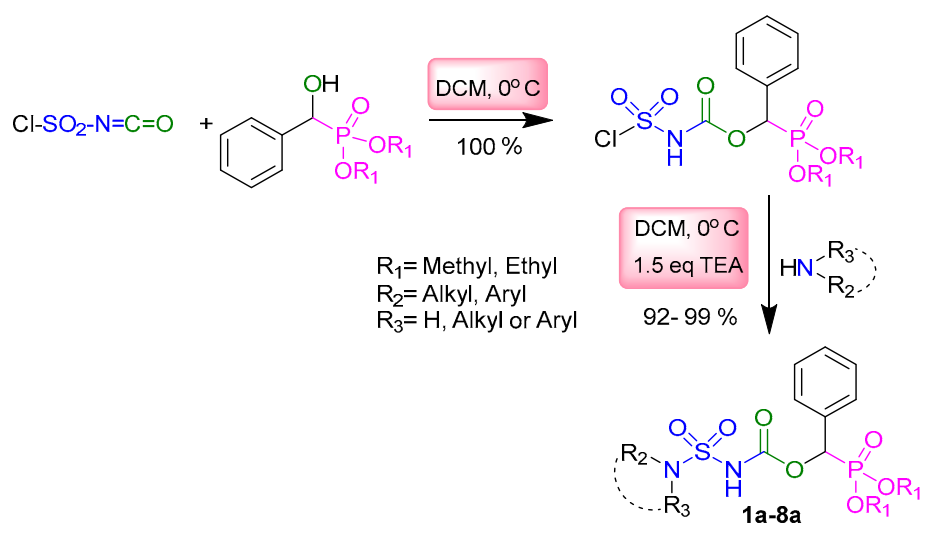

Scheme 1. Synthesis of sulfamidocarbonyloxyphosphonates 1a-8a from primary or secondary amines. 
To increase the scope of this reaction, we synthesized other sulfamidocarbonyloxyphosphonates using diverse (S)-amino acid esters (Scheme 2). The isolated yields of the products $\mathbf{1 b} \mathbf{b} \mathbf{- 3} \mathbf{b}$, obtained as a mixture of diastereoisomers (Table 2), were in the range of $84-94 \%$ yield after 90 min of reaction.

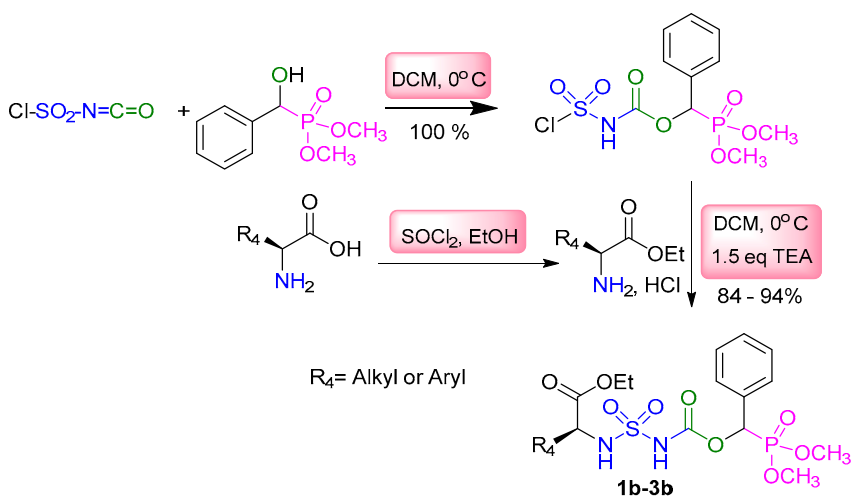

Scheme 2. Synthesis of sulfamidocarbonyloxyphosphonates $\mathbf{1} \mathbf{b}-\mathbf{3 b}$ from amino acid esters.

These satisfactory and encouraging results have prompted us to develop a third subseries (Scheme 3, Table 3), by using oxazolidin-2-one as a building block in order to synthesize new potential bioactive molecules. The new compound 1c was obtained with a very good yield (92\%).

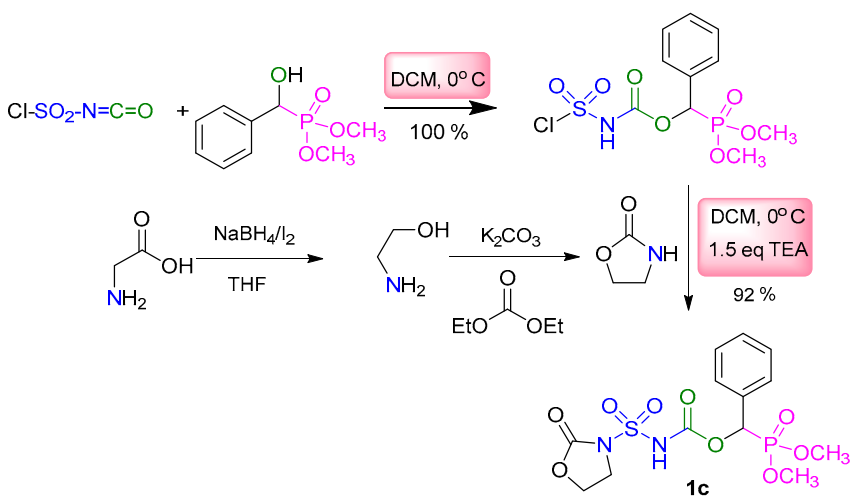

Scheme 3. Synthesis of sulfamidocarbonyloxyphosphonate 1c from oxazolidin-2-one.

Spectrometric methods confirmed the structures of all the sulfamidocarbonyloxyphosphonates synthesized. Their physicochemical and analytical data are depicted in Tables 1-3. The FT-IR spectrum showed the characteristic signals of the three functions, namely the carbamate $\mathrm{NH}$ stretching at $3300-3250 \mathrm{~cm}^{-1}$ and its $\mathrm{C}=\mathrm{O}$ stretching at $1750-1730 \mathrm{~cm}^{-1}$, the phosphonate group at $1255-1234 \mathrm{~cm}^{-1}$, and the sulfamide group with its two signals at $1185-1118 \mathrm{~cm}^{-1}$ and $1384-1356 \mathrm{~cm}^{-1}$. The molecular peak $[\mathrm{M}+\mathrm{H}]^{+}$obtained by ESI-MS was always present and corresponded to each synthesized compound. NMR spectra were recorded using $\mathrm{CDCl}_{3}$ as the solvent and are available in the supplementary material part. The ${ }^{1} \mathrm{H}$ spectrum always exhibited a dramatically deshielded doublet at $6 \mathrm{ppm}$ corresponding to the $\mathrm{COOCH}(\mathrm{Ph}) \mathrm{POOR}$ proton with its expected coupling constant ${ }^{2} J_{\mathrm{H}-\mathrm{P}}$ frequently around $12-14 \mathrm{~Hz}$. The two methoxy groups of the phosphonate appeared as two separate doublets $\left({ }^{3} \mathrm{~J}_{\mathrm{H}-\mathrm{P}} \sim 10 \mathrm{~Hz}\right)$ at 3.5 and $3.7 \mathrm{ppm}$, while the $\mathrm{NH}$ of the carbamate appeared as a broad singlet at $\delta$ 8-11 ppm. The ${ }^{13} \mathrm{C}$ spectrum was also characteristic due to the expected doublets related to the presence of the phosphorus (JC-P couplings): (i) the methoxy of the phosphonates at $54 \mathrm{ppm}$ $\left({ }^{2} J_{C-P} \sim 7-8 \mathrm{~Hz}\right)$, and (ii) the aromatic ring $\left({ }^{3} J_{\mathrm{C}-\mathrm{P} \sim 6 \mathrm{~Hz} \text { and }}{ }^{4} J_{\mathrm{C}-\mathrm{P} \sim 1-3 \mathrm{~Hz})}[35,36]\right.$. The ${ }^{13} \mathrm{C}$ chemical shifts are particular, as the carbonyl of the carbamate at $150 \mathrm{ppm}$ (doublet with a ${ }^{3} J_{\mathrm{C}-\mathrm{P}}=12 \mathrm{~Hz}$ 
coupling constant) and the greatly deshielded $\mathrm{COOCH}(\mathrm{Ph}) \mathrm{POOR}$ carbon at $70 \mathrm{ppm}$ (doublet with a ${ }^{1} J_{\mathrm{C}-\mathrm{P}}=170 \mathrm{~Hz}$ coupling constant).

To determine the initial interest of these novel functionalized sulfamidocarbonyloxyphosphonates as antibacterial agents, we only selected eight derivatives (including seven from the first sub-series) for testing their potency against sixteen bacterial strains. This first biological study can confirm the interest to modulate such a scaffold.

Table 1. The physical data and yields for sulfamidocarbonyloxyphosphonates 1a-8a synthesized from primary and secondary amines.

(1)


Table 2. Physical data and yields for sulfamidocarbonyloxyphosphonates $\mathbf{1} \mathbf{b}-\mathbf{3} \mathbf{b}$ synthesized from amino acid esters.

Entry Yield m.p. ${ }^{\circ} \mathrm{C}$

Table 3. Physical data and yield for the sulfamidocarbonyloxyphosphonate 1c synthesized from oxazolidin-2-one.

\begin{tabular}{llll}
\hline Entry & Tield \% & m.p. ${ }^{\circ} \mathrm{C}$ \\
\hline $1 c$ & & & \\
\end{tabular}

\subsection{In Vitro Antibacterial Evaluation of Sulfamidocarbonyloxyphosphonates}

A total of twelve clinical strains of Gram-positive and Gram-negative bacteria and four control strains (S. aureus ATCC 25923, E. coli ATCC 25922, P. aeruginosa ATCC 27853, and K. pneumoniae ATCC 700603) were used to investigate the antibacterial activity. The eight tested sulfamidocarbonyloxyphosphonate derivatives (compounds 1a-7a and $\mathbf{1 b}$ ) showed antibacterial activity with a varying degree of inhibitory effect on the growth of the bacterial strains (Tables 4 and 5).

The disk diffusion is just a qualitative method to determine whether a particular bacterium is susceptible to the action of a specific antimicrobial agent. The presence or the absence of a clear region around the disk is an indication of the inhibition or lack of inhibition of the bacterial growth. Then, the size of the zone of inhibition indicates the degree of sensitivity of bacteria to an antimicrobial drug. We could use the terms "resistant, intermediate, and sensitive" to discuss the results obtained. As shown in Table 4, the diameters of the inhibition zone (DIZ) of the tested compounds against the bacteria strains ranged from 12-26 mm. The values obtained with the positive control sulfamethoxazole-trimethoprim (SXT) ranged between 17 and $22 \mathrm{~mm}$ for both clinical and control strains. Furthermore, some P. aeruginosa and K. pneumoniae strains (P. aeruginosa 1, K. pneumoniae 1 and 3) were resistant (R) towards SXT. It should be noted that $P$. aeruginosa is known to be a multidrug resistant bacteria due to its remarkable ability of acquiring mechanisms of resistance to some antimicrobial agents.

Tested sulfamidocarbonyloxyphosphonate derivatives were more active toward Gram-negative bacteria than Gram-positive ones. Compound 4a was inactive on all three clinical strains of $S$. aureus 
(only DIZ = $12 \mathrm{~mm}$ for $S$. aureus ATCC 25923). Compounds $\mathbf{2 a}, \mathbf{3 a}, \mathbf{6 a}, \mathbf{7 a}$, and $\mathbf{1} \mathbf{b}$ exerted intermediate activity on $S$. aureus $(12 \leq \mathrm{DIZ} \leq 16 \mathrm{~mm}$ ). The best activities on $S$. aureus (DIZ $>16 \mathrm{~mm}$, result reported as sensitive) were observed with compounds $\mathbf{1 a}$ (S. aureus 3 ) and $\mathbf{5 a}$ (S. aureus 1 and 3 ) with zone sizes of 17,18 , and $17 \mathrm{~mm}$, respectively. Nevertheless, SXT seems to give better results, with zone sizes of between 18 and $22 \mathrm{~mm}$. On E. coli strains, all tested compounds and STX gave results with inhibition zones between 17 and $25 \mathrm{~mm}$. Among them, compounds 3a and 4a were the most active molecules against E. coli, with inhibition diameters of $25 \mathrm{~mm}$ for E. coli ATCC 25922 and $24 \mathrm{~mm}$ for E. coli 2. For P. aeruginosa strains, the inhibition zones were between 17 and $26 \mathrm{~mm}$. Their susceptibility was really marked with compounds $\mathbf{1 a}, \mathbf{4 a}$, and $\mathbf{5 a}$, with zone sizes between 18 and $26 \mathrm{~mm}$. Compound 1a showed the best activity against strains P. aeruginosa ATCC 27853 and P. aeruginosa 2, with an inhibition diameter of $26 \mathrm{~mm}$. SXT exhibited less activity against the four P. aeruginosa strains tested. For example, in the cases of P. aeruginosa ATCC 27853 and P. aeruginosa 2, the inhibition zones of STX were equal to 17 and $20 \mathrm{~mm}$, respectively. Concerning K. Pneumoniae, all tested sulfamidocarbonyloxyphosphonate derivatives were globally active, with inhibition zones superior to $15 \mathrm{~mm}$. The best activity was obtained with compound $4 \mathbf{a}$, with inhibition zones of 24 and $25 \mathrm{~mm}$ for clinical strains.

After the evidence of in vitro antibacterial activity against the tested strains in the disk diffusion test, the Minimum Inhibitory Concentration (MIC) values were determined. As shown in Table 5, most derivatives exhibited low MIC values against the different strains of bacteria employed when compared with STX $($ MIC $=25 \mu \mathrm{g} / \mathrm{mL})$. All the tested compounds showed the best MIC values against E. coli and P. aeruginosa strains, ranging between 0.5 and $32 \mu \mathrm{g} / \mathrm{mL}$. In particular, compounds 1a, 3a, and 6 a exerted the most intense activity, especially on $P$. aeruginosa, with MIC values ranging between 0.5 and $1 \mu \mathrm{g} / \mathrm{mL}$ for $\mathbf{1 a}$ and 1 and $4 \mu \mathrm{g} / \mathrm{mL}$ for $3 \mathbf{a}$ and $\mathbf{6 a}$. As regards compound $4 \mathbf{a}$, it was very active against $E$. coli strains, with MIC values between 0.5 and $4 \mu \mathrm{g} / \mathrm{mL}$. For K. pneumoniae, the best results were obtained with compounds $\mathbf{1 a}$ and $\mathbf{4 a}$, with MIC values in the range of 4 to $8 \mu \mathrm{g} / \mathrm{mL}$ and 2 to $16 \mu \mathrm{g} / \mathrm{mL}$, respectively. Concerning S. aureus strains, all MIC values were superior to $64 \mu \mathrm{g} / \mathrm{mL}$, except for compound $\mathbf{1 b}(15 \leq \mathrm{MIC} \leq 18 \mu \mathrm{g} / \mathrm{mL})$.

Overall, our results showed that the sulfamidocarbonyloxyphosphonates possessed a good concentration dependent antibacterial activity, especially against the tested Gram-negative bacteria at MIC values ranging between $0.5-32 \mu \mathrm{g} / \mathrm{mL}$ for compound $\mathbf{1 a}$, and 0.5 and $16 \mu \mathrm{g} / \mathrm{mL}$ for compound $4 \mathbf{a}$. Among the eight compounds tested, only compound $\mathbf{1 b}$ exerted antibacterial activity against $S$. aureus.

Table 4. Diameters of the inhibition zone (DIZ) of sulfamidocarbonyloxyphosphonate derivatives 1a-7a, 1b, and SXT toward Gram-positive and Gram-negative bacteria.

\begin{tabular}{|c|c|c|c|c|c|c|c|c|c|}
\hline \multirow[b]{2}{*}{ Bacterial Strains } & \multicolumn{9}{|c|}{ Diameters of Inhibition Zone (DIZ) in $\mathrm{mm}^{\text {a }}$} \\
\hline & $1 \mathbf{a}$ & $2 \mathbf{a}$ & $3 a$ & $4 a$ & $5 \mathbf{a}$ & $6 \mathbf{a}$ & $7 \mathbf{a}$ & $1 b$ & SXT \\
\hline S. aureus ATCC 25923 & 15 & 16 & 14 & 12 & 15 & 12 & 13 & 13 & 22 \\
\hline S. aureus 1 & 16 & 15 & 14 & $\mathrm{R}^{\mathrm{b}}$ & 18 & 13 & 14 & 12 & 20 \\
\hline S. aureus 2 & 16 & 15 & 16 & $\mathrm{R}$ & 16 & 15 & 12 & 13 & 18 \\
\hline S. aureus 3 & 17 & 14 & 15 & $\mathrm{R}$ & 17 & 14 & 13 & 15 & 18 \\
\hline E. coli ATCC 25922 & 24 & 23 & 25 & 25 & 23 & 21 & 23 & 18 & 20 \\
\hline E. coli 1 & 17 & 22 & 18 & 20 & 20 & 20 & 19 & 18 & 18 \\
\hline E. coli 2 & 22 & 19 & 24 & 24 & 22 & 18 & 23 & 20 & 18 \\
\hline E. coli 3 & 20 & 22 & 23 & 22 & 19 & 18 & 20 & 17 & 20 \\
\hline P. aeruginosa ATCC 27853 & 26 & 20 & 18 & 23 & 19 & 19 & 20 & 18 & 17 \\
\hline P. aeruginosa 1 & 24 & 20 & 20 & 18 & 18 & 20 & 18 & 20 & $\mathrm{R}$ \\
\hline P. aeruginosa 2 & 26 & 19 & 20 & 20 & 20 & 19 & 18 & 17 & 20 \\
\hline P. aeruginosa 3 & 25 & 21 & 21 & 22 & 22 & 21 & 19 & $\mathrm{R}$ & 18 \\
\hline $\begin{array}{l}\text { K. pneumoniae ATCC } \\
700603\end{array}$ & 22 & 19 & 13 & 20 & 19 & 20 & 21 & 19 & 22 \\
\hline K. pneumoniae 1 & 22 & 21 & 18 & 25 & 15 & 18 & 19 & 15 & $\mathrm{R}$ \\
\hline K. pneumoniae 2 & 20 & 21 & 17 & 25 & 20 & 18 & 16 & $\mathrm{R}$ & 17 \\
\hline K. pneumoniae 3 & 20 & 18 & 18 & 24 & 19 & 19 & 22 & 21 & $\mathrm{R}$ \\
\hline
\end{tabular}

${ }^{a}$ All tests were performed in triplicate. ${ }^{b}$ R: Resistant. 
Table 5. Minimum inhibitory concentrations (MICs) of the sulfamidocarbonyloxyphosphonate derivatives $\mathbf{1 a - 7 a}$ and $\mathbf{1 b}$ toward Gram-positive and Gram-negative bacteria.

\begin{tabular}{|c|c|c|c|c|c|c|c|c|}
\hline \multirow[b]{2}{*}{ Bacterial Strains } & \multicolumn{8}{|c|}{$\operatorname{MIC}(\mu \mathrm{g} / \mathrm{mL})^{a}$} \\
\hline & 1a & $2 a$ & $3 a$ & $4 \mathbf{a}$ & $5 a$ & $6 a$ & $7 a$ & $1 \mathrm{~b}$ \\
\hline S. aureus ATCC 25923 & 128 & 128 & 256 & 512 & 256 & 128 & 256 & 15 \\
\hline S. aureus 1 & 128 & 256 & 256 & $\mathrm{R}^{\mathrm{b}}$ & 64 & 128 & 256 & 15 \\
\hline S. aureus 2 & 64 & 128 & 128 & $\mathrm{R}$ & 128 & 64 & 128 & 18 \\
\hline S. aureus 3 & 64 & 128 & 128 & $\mathrm{R}$ & 128 & 128 & 128 & 15 \\
\hline E. coli ATCC 25922 & 1 & 2 & 2 & 0.5 & 2 & 2 & 8 & 18 \\
\hline E. coli 1 & 32 & 16 & 16 & 4 & 8 & 16 & 4 & 18 \\
\hline E. coli 2 & 4 & 16 & 2 & 0.5 & 4 & 16 & 8 & 20 \\
\hline E. coli 3 & 8 & 32 & 4 & 1 & 16 & 4 & 32 & 17 \\
\hline P. aeruginosa ATCC 27853 & 0.5 & 1 & 4 & 2 & 2 & 1 & 4 & 18 \\
\hline P. aeruginosa 1 & 0.5 & 2 & 2 & 4 & 2 & 2 & 2 & 20 \\
\hline P. aeruginosa 2 & 1 & 2 & 2 & 4 & 4 & 2 & 8 & 17 \\
\hline P. aeruginosa 3 & 0.5 & 6 & 1 & 2 & 2 & 4 & 4 & $\mathrm{R}$ \\
\hline K. pneumoniae АТCС 700603 & 4 & 32 & 256 & 16 & 128 & 128 & 32 & 19 \\
\hline K. pneumoniae 1 & 4 & 64 & 32 & 2 & 128 & 64 & 128 & 15 \\
\hline K. pneumoniae 2 & 8 & 16 & 16 & 2 & 32 & 128 & 128 & $\mathrm{R}$ \\
\hline K. pneumoniae 3 & 8 & 16 & 32 & 4 & 128 & 64 & 64 & 21 \\
\hline
\end{tabular}

${ }^{a}$ All tests were performed in triplicate and STX was used as the positive control (MIC $=25 \mu \mathrm{g} / \mathrm{mL}$ ). ${ }^{\mathrm{b}}$ R: Resistant.

\section{Materials and Methods}

\subsection{General Information}

All chemicals and solvents were purchased from common commercial sources and were used as received without any further purification. All reactions were monitored by TLC on silica Merck 60 F254 percolated aluminum plates and were developed by spraying with ninhydrin solution. Column chromatography was performed with Merck silica gel (230-400 mesh). Proton nuclear magnetic resonance $\left({ }^{1} \mathrm{H}\right.$ NMR) spectra were recorded on Bruker or Jeol spectrometers at $400 \mathrm{MHz}$. Chemical shifts are reported in $\delta$ units (ppm) with TMS as the reference ( $\delta 0.00)$. All coupling constants $(J)$ are reported in Hertz. Multiplicity is indicated by one or more of the following: $b$ (broad), $\mathrm{s}$ (singlet), $\mathrm{d}$ (doublet), $\mathrm{t}$ (triplet), $\mathrm{q}$ (quartet), dd (doublet of doublet), and $\mathrm{m}$ (multiplet). The Carbon nuclear magnetic resonance $\left({ }^{13} \mathrm{C}\right.$ NMR) spectra were recorded on Bruker (Reinstetten, Germany) or Jeol (JNM-ECS400 (Tokyo, Japan) spectrometers at $100.62 \mathrm{MHz}$. Chemical shifts are reported in $\delta$ units (ppm) and coupling constants $(J)$ are reported in Hertz. Phosphorus nuclear magnetic resonance $\left({ }^{31} \mathrm{P}\right.$ NMR) spectra and Fluor $\left({ }^{19} \mathrm{~F}\right.$ NMR) nuclear magnetic resonance spectra were recorded on a Bruker spectrometer at $161.98 \mathrm{MHz}$ and $316.48 \mathrm{MHz}$, respectively. Infrared spectra were recorded on a Perkin Elmer 600 (Waltham, Massachusetts, USA) spectrometer. The Mass spectra were recorded on a shimadzu QP 1100 Ex mass spectrometer operating at an ionization potential of $70 \mathrm{eV}$. Elemental analysis was recorded on a EURO E.A. 3700 apparatus. All melting points were recorded on a Büchi B-545 (Taufkirchen, Germany) apparatus in open capillary tubes.

Ultrasound assisted reactions were carried out using a FUNGILAB ultrasonic bath (Barcelona, Spain) with a frequency of $40 \mathrm{kHz}$ and a nominal power of $250 \mathrm{~W}$. The reactions were carried out in an open glass tube (diameter: $25 \mathrm{~mm}$; thickness: $1 \mathrm{~mm}$; volume: $20 \mathrm{~mL}$ ) at room temperature.

\subsection{Typical Experimental Procedure for the Synthesis of Sulfamidocarbonyloxyphosphonates $\mathbf{1 a}-\mathbf{8} \mathbf{a}, \mathbf{1} \mathbf{b}-\mathbf{3} \mathbf{b}$, and $1 \mathrm{c}$}

$\alpha$-Hydroxyphosphonates were synthesized in $94 \%$ overall yield starting from benzaldehyde and trialkylphosphites under ultrasound irradiation according to the procedure described in reference [34].

A solution of $\alpha$-hydroxyphosphonate (1.1 equiv) in anhydrous $\mathrm{CH}_{2} \mathrm{Cl}_{2}(5 \mathrm{~mL})$ was added dropwise to a stirring solution of chlorosulfonyl isocyanate (CSI) (1 equiv) in anhydrous $\mathrm{CH}_{2} \mathrm{Cl}_{2}$ $(5 \mathrm{~mL})$ at $0{ }^{\circ} \mathrm{C}$ over a period of $20 \mathrm{~min}$. The resulting solution was transferred to a mixture of primary 
or secondary amine (1.1 equiv) or amino acid ester or oxazolidin-2-one in anhydrous $\mathrm{CH}_{2} \mathrm{Cl}_{2}(10 \mathrm{~mL})$ in the presence of triethylamine (1.1-1.5 equiv). The reaction mixture was stirred at $0{ }^{\circ} \mathrm{C}$ for less than $1-2 \mathrm{~h}$, and then neutralized by adding a solution of aqueous $\mathrm{HCl} 0.1 \mathrm{M}$ to $\mathrm{pH}$. The organic layer was extracted, washed with water, dried over anhydrous sodium sulfate, filtered, and concentrated in vacuo. The pure products were crystallized in a mixture of diethyl ether $/ n$-hexane $(1.5: 1)$ at $6{ }^{\circ} \mathrm{C}$ overnight. The pure sulfamidocarbonyloxyphosphonates were finally filtered and dried in excellent yields.

(Dimethoxyphosphoryl)(phenyl)methyl (N-benzylsulfamoyl)carbamate (1a). White powder, 99\% yield, m.p. 131-133 ${ }^{\circ} \mathrm{C}, \mathrm{R}_{f}=0.43\left(\mathrm{CH}_{2} \mathrm{Cl}_{2} / \mathrm{MeOH}, 90: 10\right)$. IR $\left(\mathrm{KBr}, \mathrm{cm}^{-1}\right): 3365,3298,1733,1481,1364,1249,1170$. ${ }^{1} \mathrm{H}-\mathrm{NMR}\left(400 \mathrm{MHz}, \mathrm{CDCl}_{3}\right) \delta: 3.57\left(\mathrm{~d}, 3 \mathrm{H},{ }^{3} J_{\mathrm{H}-\mathrm{P}}=10.4 \mathrm{~Hz}, \mathrm{CH}_{3}-\mathrm{OP}\right), 3.77\left(\mathrm{~d}, 3 \mathrm{H},{ }^{3} J_{H-P}=10.8 \mathrm{~Hz}\right.$, $\left.\mathrm{CH}_{3}-\mathrm{OP}\right), 4.11\left(\mathrm{dd}, 1 \mathrm{H}, J_{1}=13.6 \mathrm{~Hz}, J_{2}=5.4 \mathrm{~Hz}, \mathrm{CH}-\mathrm{N}\right), 4.23\left(\mathrm{dd}, 1 \mathrm{H}, J_{1}=14.0 \mathrm{~Hz}, J_{2}=5.6 \mathrm{~Hz}, \mathrm{CH}-\mathrm{N}\right)$, 5.61 (bs, $\left.1 \mathrm{H}, \mathrm{NH}-\mathrm{SO}_{2}\right), 6.00\left(\mathrm{~d}, 1 \mathrm{H},{ }^{2} \mathrm{~J}_{\mathrm{H}-\mathrm{P}}=12.0 \mathrm{~Hz}, \mathrm{CH}^{*}-\mathrm{OP}\right), 7.18-7.28(\mathrm{~m}, 5 \mathrm{H}, \mathrm{H}-\mathrm{Ar}), 7.36-7.42(\mathrm{~m}, 3 \mathrm{H}$, H-Ar), 7.47-7.53 (m, 2H, H-Ar), 8.90 (bs, 1H, NH-C=O). ${ }^{13} \mathrm{C}-\mathrm{NMR}\left(100.62 \mathrm{MHz}, \mathrm{CDCl}_{3}\right) \delta: 48.15\left(\mathrm{CH}_{2}\right)$, $54.19\left(\mathrm{~d}, J_{\mathrm{C}-\mathrm{P}}=7 \mathrm{~Hz}, \mathrm{POCH}_{3}\right), 54.48\left(\mathrm{~d}, J_{\mathrm{C}-\mathrm{P}}=7 \mathrm{~Hz}, \mathrm{POCH}_{3}\right), 72.52\left(\mathrm{~d}, J_{\mathrm{C}-\mathrm{P}}=172 \mathrm{~Hz}, \mathrm{CH}^{*}-\mathrm{OP}\right), 128.03$ $\left(2 \mathrm{C}, \mathrm{d}, J_{\mathrm{C}-\mathrm{P}}=6 \mathrm{~Hz}\right), 128.18(2 \mathrm{C}), 128.34(2 \mathrm{C}), 128.54,128.96\left(2 \mathrm{C}, \mathrm{d}, J_{\mathrm{C}-\mathrm{P}}=4 \mathrm{~Hz}\right), 129.58,132.41,135.54$,

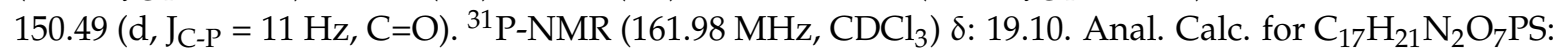
C 47.66, H 4.94, N 6.54, S 7.48. Found: C 47.71, H 4.89, N 6.52, S 7.44\%. ESI-MS: $(m / z)=429.1[\mathrm{M}+\mathrm{H}]^{+}$.

(Dimethoxyphosphoryl)(phenyl)methyl(N-(2-methoxyphenyl)sulfamoyl)carbamate (2a). White powder, $98 \%$ yield, m.p. $137-139{ }^{\circ} \mathrm{C}, \mathrm{R}_{f}=0.40\left(\mathrm{CH}_{2} \mathrm{Cl}_{2} / \mathrm{MeOH}, 90: 10\right) . \mathrm{IR}\left(\mathrm{KBr}, \mathrm{cm}^{-1}\right)$ : 3342, 3275, 1733, 1489, 1361, 1252, 1136. ${ }^{1} \mathrm{H}-\mathrm{NMR}\left(400 \mathrm{MHz}^{\mathrm{CDCl}} \mathrm{CDC}_{3}\right) \delta: 3.50\left(\mathrm{~d}, 3 \mathrm{H},{ }^{3} \mathrm{~J}_{\mathrm{H}-\mathrm{P}}=10.8 \mathrm{~Hz}, \mathrm{CH}_{3}-\mathrm{OP}\right), 3.54(\mathrm{~s}, 3 \mathrm{H}, \mathrm{CH}-\mathrm{O})$, $3.62\left(\mathrm{~d}, 3 \mathrm{H},{ }^{3} J_{H-P}=10.8 \mathrm{~Hz}, \mathrm{CH}_{3}-\mathrm{OP}\right), 5.94\left(\mathrm{~d}, 1 \mathrm{H},{ }^{2} J_{\mathrm{H}-\mathrm{P}}=14.0 \mathrm{~Hz}, \mathrm{CH}^{*}-\mathrm{OP}\right), 6.75\left(\mathrm{dd}, 1 \mathrm{H}, J_{1}=8.0 \mathrm{~Hz}\right.$, $\left.J_{2}=1.2 \mathrm{~Hz}, \mathbf{H}_{\text {ortho }}-\mathrm{Ar} \mathrm{OMe}\right), 6.84\left(\mathrm{td}, 1 \mathrm{H}, J_{1}=7.6 \mathrm{~Hz}, J_{2}=1.2 \mathrm{~Hz}, \mathbf{H}_{\text {metha }}-\mathrm{Ar}\right), 7.07\left(\mathrm{td}, 1 \mathrm{H}, J_{1}=6.8 \mathrm{~Hz}\right.$, $\left.J_{2}=1.2 \mathrm{~Hz}, \mathbf{H}-\mathrm{Ar}\right), 7.31-7.39(\mathrm{~m}, 5 \mathrm{H}, \mathrm{H}-\mathrm{Ar}), 7.43\left(\mathrm{dd}, 1 \mathrm{H}, J_{1}=8.0 \mathrm{~Hz}, J_{2}=1.6 \mathrm{~Hz}, \mathbf{H}_{\text {ortho }}\right.$-Ar NH), 7.55 (bs, 1H, NH-SO $), 9.85$ (bs, $1 \mathrm{H}, \mathrm{NH}-\mathrm{C}=\mathrm{O}) .{ }^{13} \mathrm{C}-\mathrm{NMR}\left(100.62 \mathrm{MHz}, \mathrm{CDCl}_{3}\right) \delta: 54.16\left(\mathrm{~d}, J_{\mathrm{C}-\mathrm{P}}=7 \mathrm{~Hz}\right.$, $\left.\mathrm{POCH}_{3}\right), 54.26\left(\mathrm{~d}, J_{\mathrm{C}-\mathrm{P}}=7 \mathrm{~Hz}, \mathrm{POCH}_{3}\right), 55.79\left(\mathrm{OCH}_{3}\right), 72.20\left(\mathrm{~d}, J_{\mathrm{C}-\mathrm{P}}=174 \mathrm{~Hz}, \mathrm{CH}^{*}-\mathrm{OP}\right), 111.09,120.87$, 121.04, 121.37, 125.95, $128.09\left(2 \mathrm{C}, \mathrm{d}, J_{\mathrm{C}-\mathrm{P}}=6 \mathrm{~Hz}\right), 128.86,129.33\left(2 \mathrm{C}, \mathrm{d}, J_{\mathrm{C}-\mathrm{P}}=3 \mathrm{~Hz}\right), 132.52,149.73$,

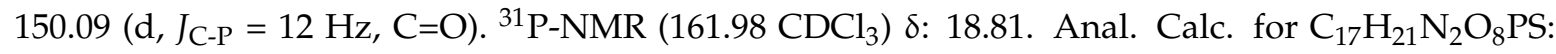
C 45.95, H 4.76, N 6.30, S 7.22. Found: C 45.90, H 4.81, N 6.28, S 7.26\%. ESI-MS: $(m / z)=445.1[\mathrm{M}+\mathrm{H}]^{+}$.

(Dimethoxyphosphoryl)(phenyl)methyl(morpholinosulfonyl)carbamate (3a). White powder, 98\% yield, m.p. 144-146 ${ }^{\circ} \mathrm{C}, \mathrm{R}_{f}=0.47\left(\mathrm{CH}_{2} \mathrm{Cl}_{2} / \mathrm{MeOH}, 90: 10\right)$. IR $\left(\mathrm{KBr}, \mathrm{cm}^{-1}\right): 3447,3297,1732,1481,1361,1247,1185$, 769, 687. ${ }^{1} \mathrm{H}-\mathrm{NMR}\left(400 \mathrm{MHz}^{\mathrm{CDCl}} \mathrm{CDC}_{3}\right) \delta: 3.29-3.31\left(\mathrm{~m}, 4 \mathrm{H}, 2 \mathrm{CH}_{2}-\mathrm{N}\right), 3.56\left(\mathrm{~d}, 3 \mathrm{H},{ }^{3} J_{H-P}=10.4 \mathrm{~Hz}\right.$, $\left.\mathrm{CH}_{3}-\mathrm{OP}\right), 3.65-3.67\left(\mathrm{~m}, 4 \mathrm{H}, 2 \mathrm{CH}_{2}-\mathrm{O}\right), 3.84\left(\mathrm{~d}, 3 \mathrm{H}^{3} J_{H-P}=10.8 \mathrm{~Hz}, \mathrm{CH}_{3}-\mathrm{OP}\right), 6.02\left(\mathrm{~d}, 1 \mathrm{H},{ }^{2} J_{H-P}=13.6 \mathrm{~Hz}\right.$, $\left.\mathrm{CH}^{*}-\mathrm{OP}\right), 7.37-7.40$ (m, 3H, H-Ar), $7.51-7.55$ (m, 2H, H-Ar), 9.92 (bs, 1H, NH-C=O). ${ }^{13} \mathrm{C}-\mathrm{NMR}$

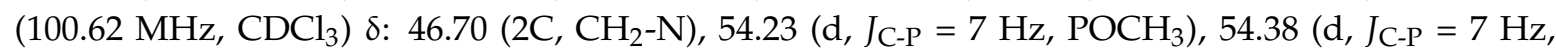
$\left.\mathrm{POCH}_{3}\right), 66.32\left(2 \mathrm{C}, \mathrm{CH}_{2}-\mathrm{O}\right), 72.09\left(\mathrm{~d}, \mathrm{~J}_{\mathrm{C}-\mathrm{P}}=174 \mathrm{~Hz}, \mathrm{CH}^{*}-\mathrm{OP}\right), 128.16\left(2 \mathrm{C}, \mathrm{d}, J_{\mathrm{C}-\mathrm{P}}=6 \mathrm{~Hz}\right), 128.92(2 \mathrm{C}, \mathrm{d}$, $\left.J_{\mathrm{C}-\mathrm{P}}=1 \mathrm{~Hz}\right), 129.56\left(\mathrm{~d}, J_{\mathrm{C}-\mathrm{P}}=3 \mathrm{~Hz}\right), 132.46,150.72\left(\mathrm{~d}, J_{\mathrm{C}-\mathrm{P}}=12 \mathrm{~Hz}, \mathrm{C}=\mathrm{O}\right) .{ }^{31} \mathrm{P}-\mathrm{NMR}\left(161.98 \mathrm{CDCl}_{3}\right) \delta$ : 18.93. Anal. Calc. for $\mathrm{C}_{14} \mathrm{H}_{21} \mathrm{~N}_{2} \mathrm{O}_{8}$ PS: C 41.18, H 5.18, N 6.86, S 7.85. Found: C 41.22, H 5.23, N 6.83, S 7.81\%. ESI-MS: $(m / z)=409.1[\mathrm{M}+\mathrm{H}]^{+}$.

(Dimethoxyphosphoryl)(phenyl)methyl(N-(3-fluorophenyl)sulfamoyl)carbamate (4a). White powder, 96\% yield, m.p. $136-138{ }^{\circ} \mathrm{C}, \mathrm{R}_{f}=0.41\left(\mathrm{CH}_{2} \mathrm{Cl}_{2} / \mathrm{MeOH}, 90: 10\right) . \mathrm{IR}\left(\mathrm{KBr}, \mathrm{cm}^{-1}\right): 3311,3297,1758,1477,1355$, 1251, 1166. ${ }^{1} \mathrm{H}-\mathrm{NMR}\left(400 \mathrm{MHz} \mathrm{CDCl}_{3}\right) \delta: 3.62\left(\mathrm{dd}, 3 \mathrm{H}, J_{1}=38.8 \mathrm{~Hz}, J_{2}=10.4 \mathrm{~Hz}, \mathrm{CH}_{3}-\mathrm{O}\right), 3.72(\mathrm{dd}, 3 \mathrm{H}$, $\left.J_{1}=10.4 \mathrm{~Hz}, J_{2}=1.2 \mathrm{~Hz}, \mathrm{CH}_{3}-\mathrm{O}\right), 5.95\left(\mathrm{~d}, 1 \mathrm{H}, J=13.6, \mathrm{CH}^{*}-\mathrm{O}\right), 6.88-7.04(\mathrm{~m}, 3 \mathrm{H}, \mathbf{H}-\mathrm{Ar}), 7.19-7.41(\mathrm{~m}$, $6 \mathrm{H}, \mathrm{H}-\mathrm{Ar}) .{ }^{13} \mathrm{C}-\mathrm{NMR}\left(100.62 \mathrm{MHz}, \mathrm{CDCl}_{3}\right) \delta: 54.85,54.90,71.86,73.21,128.14,128.30,129.23,129.65$, 12.91, 131.15, 131.75, 134.19, 134.56, 138.25, 138.45, 150.36. ${ }^{31} \mathrm{P}-\mathrm{NMR}\left(161.98 \mathrm{CDCl}_{3}\right)$ 20.61. ${ }^{19} \mathrm{~F}-\mathrm{NMR}$ $\left(316.48 \mathrm{MHz}_{2} \mathrm{CDCl}_{3}\right.$ ) $\delta$ : -111.62. Anal. Calc. for $\mathrm{C}_{16} \mathrm{H}_{18} \mathrm{FN}_{2} \mathrm{O}_{7} \mathrm{PS}$ : C 44.45, $\mathrm{H} 4.20, \mathrm{~N}$ 6.48, S 7.42. Found: C 44.40, H 4.23, N 6.52, S 7.41\%. ESI-MS: $(m / z)=433.1[\mathrm{M}+\mathrm{H}]^{+}$.

(Diethoxyphosphoryl)(phenyl)methyl(N-phenylsulfamoyl)carbamate (5a). White powder, 96\% yield, m.p. 187-189 ${ }^{\circ} \mathrm{C}, \mathrm{R}_{f}=0.42\left(\mathrm{CH}_{2} \mathrm{Cl}_{2} / \mathrm{MeOH}, 90: 10\right)$. IR $\left(\mathrm{KBr}, \mathrm{cm}^{-1}\right): 3447,3297,1733,1481,1384,1247,1185$. $\left.{ }^{1} \mathrm{H}-\mathrm{NMR}\left(400 \mathrm{MHz}^{\mathrm{CDCl}}\right)_{3}\right) \delta: 1.03\left(\mathrm{t}, 3 \mathrm{H}, J=7.0 \mathrm{~Hz}, \mathrm{CH}_{3}\right), 1.30\left(\mathrm{t}, 3 \mathrm{H}, J=7.0 \mathrm{~Hz}, \mathrm{CH}_{3}\right), 3.59-3.68(\mathrm{~m}$, 
$\left.1 \mathrm{H}, \mathrm{CH}_{2}-\mathrm{O}\right), 3.82-3.90\left(\mathrm{~m}, 1 \mathrm{H}, \mathrm{CH}_{2}-\mathrm{O}\right), 4.07-4.17\left(\mathrm{~m}, 2 \mathrm{H}, \mathrm{CH}_{2}-\mathrm{O}\right), 5.82\left(\mathrm{~d}, 1 \mathrm{H}, J=8.8 \mathrm{~Hz}, \mathrm{CH}^{*} \mathrm{OP}\right), 6.47$ $\left(\mathrm{s}, 1 \mathrm{H}, \mathrm{NH}-\mathrm{SO}_{2}\right), 6.80\left(\mathrm{dd}, 2 \mathrm{H}, J_{1}=8.8 \mathrm{~Hz}, J_{2}=1.2 \mathrm{~Hz}, \mathrm{H}-\mathrm{Ar}\right), 7.02(\mathrm{t}, 1 \mathrm{H}, J=7.6 \mathrm{~Hz}, \mathrm{H}-\mathrm{Ar}), 7.15(\mathrm{t}$, $2 \mathrm{H}, J=7.6 \mathrm{~Hz}, \mathrm{H}-\mathrm{Ar}), 7.20-7.26(\mathrm{~m}, 5 \mathrm{H}, \mathrm{H}-\mathrm{Ar}) .{ }^{13} \mathrm{C}-\mathrm{NMR}\left(100.62 \mathrm{MHz}, \mathrm{CDCl}_{3}\right) \delta: 16.32\left(\mathrm{CH}_{3}\right), 16.59$ $\left(\mathrm{CH}_{3}\right), 63.96\left(\mathrm{CH}_{2}\right), 64.10\left(\mathrm{CH}_{2}\right), 72.46\left(\mathrm{~d}, J_{\mathrm{C}-\mathrm{P}}=170 \mathrm{~Hz}, \mathrm{CH}^{*}-\mathrm{OP}\right), 119.77(2 \mathrm{C}), 124.46,128.31(2 \mathrm{C}, \mathrm{d}$, $\left.J_{\mathrm{C}-\mathrm{P}}=6 \mathrm{~Hz}\right), 128.76(2 \mathrm{C}), 128.90(2 \mathrm{C}), 129.32,134.25,136.86,150.40\left(\mathrm{~d}, J_{\mathrm{C}-\mathrm{P}}=16 \mathrm{~Hz}, \mathrm{C}=\mathrm{O}\right) .{ }^{31} \mathrm{P}-\mathrm{NMR}$ $\left(161.98 \mathrm{MHz}, \mathrm{CDCl}_{3}\right)$ 8: 19.61. Anal. Calc. for $\mathrm{C}_{18} \mathrm{H}_{23} \mathrm{~N}_{2} \mathrm{O}_{7} \mathrm{PS}$ : C 48.87, H 5.24, N 6.33, S 7.25. Found: C 48.93, H 5.21, N 6.28, S 7.26\%. ESI-MS: $(m / z)=443.1[\mathrm{M}+\mathrm{H}]^{+}$.

(Dimethoxyphosphoryl)(phenyl)methyl(3,4-dihydroisoquinolin-2(1H)-yl)sulfonylcarbamate (6a). Color powder, 94\% yield, m.p. $153-155{ }^{\circ} \mathrm{C}, \mathrm{R}_{f}=0.49\left(\mathrm{CH}_{2} \mathrm{Cl}_{2} / \mathrm{MeOH}, 90: 10\right) . \mathrm{IR}\left(\mathrm{KBr}, \mathrm{cm}^{-1}\right): 3258,1750,1360$, 1454, 1234, 1120. ${ }^{1} \mathrm{H}-\mathrm{NMR}\left(400 \mathrm{MHz}, \mathrm{CDCl}_{3}\right) \delta: 2.87\left(\mathrm{t}, 2 \mathrm{H}, J=6.0 \mathrm{~Hz}, \mathrm{C}_{\mathrm{Ar}}-\mathrm{CH}_{2}-\mathrm{CH}_{2}\right), 3.55\left(\mathrm{~d}, 3 \mathrm{H},{ }^{3} J_{\mathrm{H}-\mathrm{P}}\right.$ $\left.=10.0 \mathrm{~Hz}, \mathrm{CH}_{3}-\mathrm{OP}\right), 3.60\left(\mathrm{t}, 2 \mathrm{H}, J=6.0 \mathrm{~Hz}, \mathrm{CH}_{2}-\mathrm{CH}_{2}-\mathrm{N}\right), 3.75\left(\mathrm{~d}, 3 \mathrm{H},{ }^{3} J_{\mathrm{H}-\mathrm{P}}=10.0 \mathrm{~Hz}, \mathrm{CH}_{3}-\mathrm{O}\right), 4.52(\mathrm{~s}$, $\left.2 \mathrm{H}, \mathrm{C}_{\mathrm{Ar}}-\mathrm{CH}_{2}-\mathrm{N}\right), 6.00\left(\mathrm{~d}, 1 \mathrm{H},{ }^{2} J_{\mathrm{H}-\mathrm{P}}=12.0 \mathrm{~Hz}, \mathrm{CH}^{*}-\mathrm{O}\right), 7.01-7.08(\mathrm{~m}, 2 \mathrm{H}, \mathbf{H}-\mathrm{Ar}), 7.14-7.16(\mathrm{~m}, 2 \mathrm{H}, \mathbf{H}-\mathrm{Ar})$, 7.33-7.36 (m, 3H, H-Ar), 7.48-7.51 (m, 2H, H-Ar), 9.91 (s, 1H, NH-C=O). ${ }^{13} \mathrm{C}-\mathrm{NMR}\left(100.62 \mathrm{MHz}, \mathrm{CDCl}_{3}\right)$

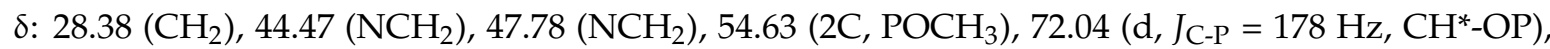
126.4, 126.6, 127.1, 128.6 (2C), 128.8, 129.60 (2C), 129.80, 131.41, 132.4, 133.2, 150.95 (d, JC-P $=16 \mathrm{~Hz}$, $\mathrm{C}=\mathrm{O}) .{ }^{31} \mathrm{P}-\mathrm{NMR}\left(161.98 \mathrm{MHz}, \mathrm{CDCl}_{3}\right)$ ): 18.76. Anal. Calc. for $\mathrm{C}_{19} \mathrm{H}_{23} \mathrm{~N}_{2} \mathrm{O}_{7} \mathrm{PS}: \mathrm{C} 50.22, \mathrm{H}$ 5.10, $\mathrm{N}$ 6.10, S 7.06. Found: C 50.19, H 5.15, N 6.16, S 7.10\%. ESI-MS: $(m / z)=453.2[\mathrm{M}-\mathrm{H}]^{+}$.

(Dimethoxyphosphoryl)(phenyl)methyl(4-phenylpiperazin-1-yl)sulfonylcarbamate (7a). White powder, 93\% yield, m.p. $152-154{ }^{\circ} \mathrm{C}, \mathrm{R}_{f}=0.50\left(\mathrm{CH}_{2} \mathrm{Cl}_{2} / \mathrm{MeOH}, 90: 10\right)$. IR $\left(\mathrm{KBr}, \mathrm{cm}^{-1}\right): 3337,1741,1449,1360,1248$, 1167. ${ }^{1} \mathrm{H}-\mathrm{NMR}\left(400 \mathrm{MHz}, \mathrm{CDCl}_{3}\right) \delta: 3.10-3.40\left(\mathrm{~m}, 4 \mathrm{H}, 2 \mathrm{CH}_{2}-\mathrm{N}_{-} \mathrm{SO}_{2}\right), 3.42-3.62\left(\mathrm{~m}, 4 \mathrm{H}, 2 \mathrm{CH}_{2}-\mathrm{N}-\mathrm{C}_{\mathrm{Ar}}\right)$, $3.67\left(\mathrm{~d}, 3 \mathrm{H}, J=10.6 \mathrm{~Hz}, \mathrm{CH}_{3}-\mathrm{O}\right), 3.75\left(\mathrm{~d}, 3 \mathrm{H}, J=10.8 \mathrm{~Hz}, \mathrm{CH}_{3}-\mathrm{O}\right), 6.05\left(\mathrm{~d}, 1 \mathrm{H},{ }^{2} J_{\mathrm{H}-\mathrm{P}}=14.0 \mathrm{~Hz}, \mathrm{CH}^{*}-\mathrm{O}\right)$, 6.80-6.96 (m, 3H, H-Ar), 7.25-7.40 (m, 5H, H-Ar), 7.45-7.56 (m, 2H, H-Ar). ${ }^{13} \mathrm{C}-\mathrm{NMR}(100.62 \mathrm{MHz}$, $\left.\mathrm{CDCl}_{3}\right) \delta: 46.78\left(2 \mathrm{C}, \mathrm{NCH}_{2}\right), 48.90\left(2 \mathrm{C}, \mathrm{NCH}_{2}\right), 54.61\left(\mathrm{~d}, J_{\mathrm{C}-\mathrm{P}}=7 \mathrm{~Hz}, \mathrm{POCH}_{3}\right), 54.62\left(\mathrm{~d}, J_{\mathrm{C}-\mathrm{P}}=7 \mathrm{~Hz}\right.$, $\left.\mathrm{POCH}_{3}\right), 70.86\left(\mathrm{~d}, J_{\mathrm{C}-\mathrm{P}}=142.4 \mathrm{~Hz}, \mathrm{CH}^{*}-\mathrm{OP}\right), 117.37(2 \mathrm{C}), 120.93,127.87\left(2 \mathrm{C}, \mathrm{d}, J_{\mathrm{C}-\mathrm{P}}=5 \mathrm{~Hz}\right), 128.57$, $128.92(2 \mathrm{C}), 129.65(2 \mathrm{C}), 132.49,136.81,151.27\left(\mathrm{~d}, J_{\mathrm{C}-\mathrm{P}}=12 \mathrm{~Hz}, \mathrm{C}=\mathrm{O}\right) .{ }^{31} \mathrm{P}-\mathrm{NMR}\left(161.98 \mathrm{MHz}, \mathrm{CDCl}_{3}\right) \delta$ : 19.82. Anal. Calc. for $\mathrm{C}_{20} \mathrm{H}_{26} \mathrm{~N}_{3} \mathrm{O}_{7} \mathrm{PS}$ : C 49.68, H 5.42, N 8.69, $\mathrm{S}$ 6.63. Found: C 49.73, H 5.46, $\mathrm{N} 8.65$, S 6.67\%. ESI-MS: $(m / z)=482.3[\mathrm{M}-\mathrm{H}]^{+}$.

(Diethoxyphosphoryl)(phenyl)methyl(N-propylsulfamoyl)carbamate (8a). White powder, 97\% yield, m.p. 151-153 ${ }^{\circ} \mathrm{C}, \mathrm{R}_{f}=0.43\left(\mathrm{CH}_{2} \mathrm{Cl}_{2} / \mathrm{MeOH}, 90: 10\right) . \mathrm{IR}\left(\mathrm{KBr}, \mathrm{cm}^{-1}\right): 3369,3061,1758,1475,1355,1240$, 1156, 763, 697. ${ }^{1} \mathrm{H}-\mathrm{NMR}\left(400 \mathrm{MHz}, \mathrm{CDCl}_{3}\right) \delta: 0.72\left(\mathrm{t}, 3 \mathrm{H}, J=8.8 \mathrm{~Hz}, \mathrm{CH}_{3}-\mathrm{Pr}\right), 1.09(\mathrm{t}, 3 \mathrm{H}, J=9.4 \mathrm{~Hz}$, $\mathrm{CH}_{3}$-OEt), 1.12-1.27 (m, 2H, CH $\left.\mathbf{H}_{2}-\mathrm{Pr}\right), 1.37$ (t, 3H, J = 9.4 Hz, $\left.\mathrm{CH}_{3}-\mathrm{OEt}\right), 2.48-2.59\left(\mathrm{~m}, 1 \mathrm{H}, \mathrm{CH}_{2}-\mathrm{N}\right)$, 2.78-2.87 (m, 1H, CH $\left.\mathrm{CH}_{2}-\mathrm{N}\right), 3.67-4.05\left(\mathrm{~m}, 2 \mathrm{H}, \mathrm{CH}_{2}-\mathrm{OP}\right), 4.25(1 \mathrm{H}, \mathrm{m}, \mathrm{NH}), 4.74\left(\mathrm{dq}, 2 \mathrm{H},{ }^{3} J_{H-P}=11.8 \mathrm{~Hz}\right.$, $\left.{ }^{3} J_{\mathrm{H}-\mathrm{H}}=7.5 \mathrm{~Hz}, \mathrm{CH}_{2}-\mathrm{OP}\right), 6.00\left(\mathrm{dd}, 1 \mathrm{H},{ }^{2} J_{\mathrm{H}-\mathrm{P}}=11.3 \mathrm{~Hz}, J=8.8 \mathrm{~Hz}, \mathrm{CH}^{*}-\mathrm{O}\right), 7.35-7.38(\mathrm{~m}, 3 \mathrm{H}, \mathbf{H}-\mathrm{Ar})$, 7.50-7.52 (m, 2H, H-Ar). Anal. Calc. for $\mathrm{C}_{15} \mathrm{H}_{25} \mathrm{~N}_{2} \mathrm{O}_{7} \mathrm{PS}$ : C 44.11, H 6.17, $\mathrm{N}$ 6.86, S 7.85. Found: C 44.29, H 6.79, N 6.91, S 7.80\%. ESI-MS: $(m / z)=409.2[\mathrm{M}+\mathrm{H}]^{+}$.

(SR) and (SS)-Ethyl-2-((N-((dimethoxyphosphoryl)(phenyl)methoxy)carbonyl)sulfamoyl)amino) -4-methylpentanoate (1b). White powder, 91\% yield; m.p. $118-120^{\circ} \mathrm{C}, \mathrm{R}_{f}=0.39\left(\mathrm{CH}_{2} \mathrm{Cl}_{2} / \mathrm{MeOH}, 90: 10\right)$. IR $\left(\mathrm{KBr}, \mathrm{cm}^{-1}\right): 3274,1747$ (l), 1470, 1371, 1251, 1164. ${ }^{1} \mathrm{H}-\mathrm{NMR}\left(400 \mathrm{MHz}, \mathrm{CDCl}_{3}\right)$ 8: 0.81-0.87 (m, $\left.12 \mathrm{H}, \mathrm{CH}_{3}-\mathrm{CH}_{\text {isop }}\right), 1.05-1.35\left(\mathrm{~m}, 6 \mathrm{H}, \mathrm{O}-\mathrm{CH}_{2}-\mathrm{CH}_{3}\right), 1.36-1.60\left(\mathrm{~m}, 4 \mathrm{H}, 2 \mathrm{CH}_{\text {isop }}+1 \mathrm{CH}_{2}-\mathrm{CH}_{\text {isop }}\right), 1.20$ $\left(\mathrm{m}, 2 \mathrm{H}, 1 \mathrm{CH}_{2}-\mathrm{CH}_{\text {isop }}\right), 3.51\left(\mathrm{~d}, 3 \mathrm{H}, J=10.6 \mathrm{~Hz}, \mathrm{CH}_{3}-\mathrm{O}\right), 3.52\left(\mathrm{~d}, 3 \mathrm{H}, J=10.6 \mathrm{~Hz}, \mathrm{CH}_{3}-\mathrm{O}\right), 3.60-3.75$ $\left(\mathrm{m}, 1 \mathrm{H}, \mathbf{C H}^{*}-\mathrm{NH}\right), 3.75-3.99\left(\mathrm{~m}, 3 \mathrm{H},-\mathrm{O}-\mathbf{C H}_{2}-\mathrm{CH}_{3}+\mathbf{C H}^{*}-\mathrm{NH}\right), 3.78\left(\mathrm{~d}, 6 \mathrm{H}, J=10.8 \mathrm{~Hz}, \mathrm{CH}_{3}-\mathrm{O}\right)$, 4.00-4.25 (m, 2H, $\left.-\mathrm{O}-\mathrm{CH}_{2}-\mathrm{CH}_{3}\right), 5.79\left(\mathrm{bs}, 1 \mathrm{H}, \mathrm{NH}-\mathrm{SO}_{2}\right), 5.96\left(\mathrm{~d}, 1 \mathrm{H}, J=13.9 \mathrm{~Hz}, \mathrm{CH}^{*}-\mathrm{O}\right), 6.00(\mathrm{~d}, 1 \mathrm{H}$, $J=14.3 \mathrm{~Hz}, \mathrm{CH}^{*}-\mathrm{O}$ ), 6.21 (bs, $1 \mathrm{H}, \mathrm{NH}^{-\mathrm{SO}_{2}}$ ), 7.32-7.40 (m, 6H, H-Ar), 7.50-7.56 (m, 4H, H-Ar), 9.86 (bs, 1H, NH-C=O). ${ }^{13} \mathrm{C}-\mathrm{NMR}\left(100.62 \mathrm{MHz}, \mathrm{CDCl}_{3}\right) \delta: 14.04\left(2 \mathrm{CH}_{3}\right), 22.76(4 \mathrm{C}), 24.37(2 \mathrm{C}), 41.95(2 \mathrm{C})$, $54.19\left(2 \mathrm{POCH}_{3}\right), 54.44\left(2 \mathrm{POCH}_{3}\right), 55.55(2 \mathrm{C}), 61.63\left(2 \mathrm{OCH}_{2}\right), 72.13\left(2 \mathrm{C}, \mathrm{d}, J_{\mathrm{C}-\mathrm{P}}=142.4 \mathrm{~Hz}, \mathrm{CH}^{*}-\mathrm{OP}\right)$, $128.03(4 \mathrm{C}), 128.12(2 \mathrm{C}), 128.75\left(4 \mathrm{C}, \mathrm{d}, J_{\mathrm{C}-\mathrm{P}}=5 \mathrm{~Hz}\right), 132.50(2 \mathrm{C}), 150.60\left(2 \mathrm{C}, \mathrm{d}, J_{\mathrm{C}-\mathrm{P}}=2 \mathrm{~Hz}, \mathrm{C}=\mathrm{O}\right), 172.01$ $(2 \mathrm{C}=\mathrm{O}) .{ }^{31} \mathrm{P}-\mathrm{NMR}\left(161.98 \mathrm{MHz}, \mathrm{CDCl}_{3}\right)$ 8: 21.61. Anal. Calc. for $\mathrm{C}_{18} \mathrm{H}_{29} \mathrm{~N}_{2} \mathrm{O}$ PS: C 45.00, H 6.08, N 5.83, S 6.67. Found: C 45.07, H 6.04, N 5.81, S 6.72\%. ESI-MS: $(m / z)=481.1[\mathrm{M}+\mathrm{H}]^{+}$. 
(SR) and (SS)-Ethyl-2-((N-((dimethoxyphosphoryl)(phenyl)methoxy)carbonyl)sulfamoyl)amino) -3-phenylpropanoate (2b). White powder, 94\% yield; m.p. $125-127{ }^{\circ} \mathrm{C}, \mathrm{R}_{f}=0.41\left(\mathrm{CH}_{2} \mathrm{Cl}_{2} / \mathrm{MeOH}\right.$ 90:10). IR (KBr, cm $\left.{ }^{-1}\right): 3279,1744(1), 1455,1373,1249,1162 .{ }^{1} \mathrm{H}-\mathrm{NMR}\left(400 \mathrm{MHz}, \mathrm{CDCl}_{3}\right) \delta: 1.01(\mathrm{t}$, $\left.3 \mathrm{H}, J=7.6 \mathrm{~Hz}, \mathrm{CH}_{3}-\mathrm{CH}_{2-} \mathrm{O}\right), 1.02\left(\mathrm{t}, 3 \mathrm{H}, J=7.6 \mathrm{~Hz}, \mathrm{CH}_{3}-\mathrm{CH}_{2-} \mathrm{O}\right), 2.85-3.15(\mathrm{~m}, 4 \mathrm{H}, \mathrm{CH} 2-\mathrm{Ar}), 3.49$ (d, $\left.6 \mathrm{H}, J=9.2 \mathrm{~Hz}, \mathrm{CH}_{3}-\mathrm{O}\right), 3.82\left(\mathrm{~d}, 6 \mathrm{H}, \mathrm{J}=9.6 \mathrm{~Hz}, \mathrm{CH}_{3}-\mathrm{O}\right), 3.75-4.00\left(\mathrm{~m}, 3 \mathrm{H}, \mathrm{CH}-\mathrm{NH}+-\mathrm{O}-\mathrm{CH}_{2}-\mathrm{CH}_{3}\right)$, 4.09-4.20 (m, 1H, CH*-NH), 4.25-4.50 (m, 3H, -O-CH $\left.-\mathrm{CH}_{3}+\mathrm{NH}-\mathrm{SO}_{2}\right), 4.86\left(\mathrm{~s}, 1 \mathrm{H}, \mathrm{NH}-\mathrm{SO}_{2}\right), 5.97$ $\left(\mathrm{d}, 1 \mathrm{H}, J=13.5 \mathrm{~Hz}, \mathrm{CH}^{*}-\mathrm{O}\right), 5.98\left(\mathrm{~d}, 1 \mathrm{H}, J=14.3 \mathrm{~Hz}, \mathrm{CH}^{*}-\mathrm{O}\right), 7.00-7.12$ (m, 2H, H-Ar), 7.11-7.41 (m, 14H, H-Ar), 7.42-7.48 (m, 4H, H-Ar). ${ }^{13} \mathrm{C}-\mathrm{NMR}\left(100.62 \mathrm{MHz}, \mathrm{CDCl}_{3}\right) \delta: 13.98\left(2 \mathrm{CH}_{3}\right), 38.99\left(2 \mathrm{CH}_{2}\right)$, $54.27\left(2 \mathrm{C}, \mathrm{d}, J_{\mathrm{C}-\mathrm{P}}=6.9 \mathrm{~Hz}, \mathrm{POCH}_{3}\right), 54.45\left(2 \mathrm{C}, \mathrm{d}, J_{\mathrm{C}-\mathrm{P}}=6.9 \mathrm{~Hz}, \mathrm{POCH}_{3}\right), 57.74(2 \mathrm{CH}), 61.76\left(2 \mathrm{OCH}_{2}\right)$, $71.14\left(2 \mathrm{C}, \mathrm{d}, J_{\mathrm{C}-\mathrm{P}}=155.6 \mathrm{~Hz}, \mathrm{CH}^{*}-\mathrm{OP}\right), 128.06\left(4 \mathrm{C}, \mathrm{d}, J_{\mathrm{C}-\mathrm{P}}=6 \mathrm{~Hz}\right), 128.09(4 \mathrm{C}), 128.57$ (4C), 128.79 (4C), $129.51\left(4 \mathrm{C}, \mathrm{d}, J_{\mathrm{C}-\mathrm{P}}=6 \mathrm{~Hz}\right), 132.2(2 \mathrm{C}), 135.5(2 \mathrm{C}), 150.69(2 \mathrm{C}=\mathrm{O}), 170.66(2 \mathrm{C}=\mathrm{O}) .{ }^{31} \mathrm{P}-\mathrm{NMR}(161.98 \mathrm{MHz}$,

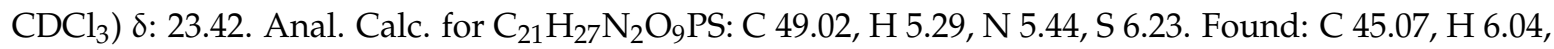
N 5.81, S 6.72\%. ESI-MS: $(m / z)=515.21[\mathrm{M}+\mathrm{H}]^{+}$.

(SR) and (SS)-Ethyl-2-((N-(((dimethoxyphosphoryl)(phenyl)methoxy)carbonyl)sulfamoyl) amino)-3-(1H-indol-3-yl) propanoate (3b). White powder, 84\% yield; m.p. $116-118{ }^{\circ} \mathrm{C} ; \mathrm{R}_{f}=0.39$ $\left(\mathrm{CH}_{2} \mathrm{Cl}_{2} / \mathrm{MeOH}, 90: 10\right)$. IR $\left(\mathrm{KBr}, \mathrm{cm}^{-1}\right): 3274,1747,1471,1371,1250,1164 .{ }^{1} \mathrm{H}-\mathrm{NMR}\left(400 \mathrm{MHz}, \mathrm{CDCl}_{3}\right)$ $\delta: 0.99\left(\mathrm{t}, 3 \mathrm{H}, \mathrm{J}=7.20 \mathrm{~Hz}, \mathrm{CH}_{3}-\mathrm{CH}_{2-} \mathrm{O}\right), 1.06\left(\mathrm{t}, 3 \mathrm{H}, J=7.2 \mathrm{~Hz}, \mathrm{CH}_{3}-\mathrm{CH}_{2-} \mathrm{O}\right), 3.11(\mathrm{~d}, 4 \mathrm{H}, \mathrm{J}=6.0 \mathrm{~Hz}$, $\left.\mathrm{CH}_{2}-\mathrm{CH}^{*}\right), 3.30-3.50\left(\mathrm{~m}, 8 \mathrm{H}, 2 \mathrm{CH}_{3}-\mathrm{O}+2 \mathrm{CH}^{*} \mathrm{CO}\right), 3.82-4.00\left(\mathrm{~m}, 8 \mathrm{H}, 2 \mathrm{CH}_{3}-\mathrm{O}+\mathrm{OCH}\right)_{2}, 4.21-4.26$ $\left(\mathrm{m}, 2 \mathrm{H}, \mathrm{OCH}_{2}\right), 6.20\left(\mathrm{~d}, 2 \mathrm{H}, J=7.8 \mathrm{~Hz}, \mathrm{CH}^{*}-\mathrm{O}\right), 6.68-6.98(\mathrm{~m}, 6 \mathrm{H}, \mathbf{H}-\mathrm{Ar}), 7.18-7.26$ (m, 8H, H-Ar),

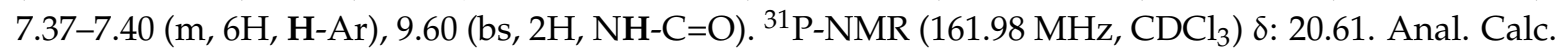
for $\mathrm{C}_{23} \mathrm{H}_{28} \mathrm{~N}_{3} \mathrm{O}_{9} \mathrm{PS}$ : C 49.91, H 5.10, N 7.59, S 5.79. Found: C 49.97, H 5.04, N 7.68, S 5.83\%. ESI-MS: $(m / z)=553.21[\mathrm{M}]^{+}$.

(Dimethoxyphosphoryl)(phenyl)methyl ((2-oxooxazolidin-3-yl)sulfonyl)carbamate (1c). White powder; 92\% yield; m.p. $123-125^{\circ} \mathrm{C} ; \mathrm{R}_{f}=0.38\left(\mathrm{CH}_{2} \mathrm{Cl}_{2} / \mathrm{MeOH}, 90: 10\right) . \mathrm{IR}\left(\mathrm{KBr}, \mathrm{cm}^{-1}\right): 3255,1748,1663,1357,1254$, 1118, 757, 629; ${ }^{1} \mathrm{H}-\mathrm{NMR}\left(400 \mathrm{MHz}, \mathrm{CDCl}_{3}\right) \delta: 3.40-3.43\left(\mathrm{~m}, 2 \mathrm{H}, \mathrm{CH}_{2}-\mathrm{N}\right), 3.61\left(\mathrm{~d}, 3 \mathrm{H},{ }^{3} J_{H-P}=8.0 \mathrm{~Hz}\right.$, $\left.\mathrm{CH}_{3}-\mathrm{OP}\right), 3.75\left(\mathrm{~d}, 3 \mathrm{H},{ }^{3} J_{H-P}=8.0 \mathrm{~Hz}, \mathrm{CH}_{3}-\mathrm{OP}\right), 4.60-4.63\left(\mathrm{~m}, 2 \mathrm{H}, \mathrm{CH}_{2}-\mathrm{O}\right), 6.04\left(\mathrm{~d}, 1 \mathrm{H},{ }^{2} J_{H-P}=12.0 \mathrm{~Hz}\right.$, $\mathrm{CH}^{*}$-OP), 7.31-7.35 (m, 3H, H-Ar), 7.37-7.39 (m, 2H, H-Ar). ${ }^{13} \mathrm{C}-\mathrm{NMR}\left(100.62 \mathrm{MHz}, \mathrm{CDCl}_{3}\right) \delta: 46.58$, $54.92\left(\mathrm{~d}, J_{\mathrm{C}-\mathrm{P}}=7 \mathrm{~Hz}, \mathrm{POCH}_{3}\right), 54.95\left(\mathrm{~d}, J_{\mathrm{C}-\mathrm{P}}=7 \mathrm{~Hz}, \mathrm{POCH}_{3}\right), 70.76,71.02\left(\mathrm{~d}, J_{\mathrm{C}-\mathrm{P}}=171 \mathrm{~Hz}, \mathrm{CH}\right.$-OP), $127.93\left(2 \mathrm{C}, \mathrm{d}, J_{\mathrm{C}-\mathrm{P}}=3 \mathrm{~Hz}\right), 128.75,128.99\left(2 \mathrm{C}, \mathrm{d}, J_{\mathrm{C}-\mathrm{P}}=2 \mathrm{~Hz}\right), 133.52,155.06(\mathrm{C}=\mathrm{O}), 155.12\left(\mathrm{~d}, J_{\mathrm{C}-\mathrm{P}}=12 \mathrm{~Hz}\right.$,

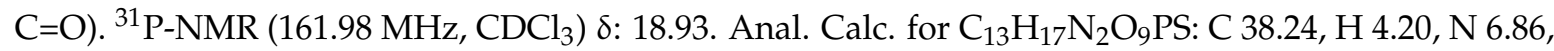
S 7.85. Found: C 38.20, H 4.25, N 6.89, S 7.81\%. ESI-MS: $(m / z)=431.5[\mathrm{M}+\mathrm{Na}]^{+}$.

\subsection{Determination of In Vitro Antibacterial Activity}

The antimicrobial activity of the synthesized compounds was evaluated in vitro against Gram positive and Gram negative bacteria. Serial dilutions of the tested compounds in acetone were made in a concentration range from 0.5 to $512 \mu \mathrm{g} / \mathrm{mL}$. All tests were performed in triplicate.

Firstly, compounds $\mathbf{1 a}-\mathbf{7} \mathbf{a}$ and $\mathbf{1 b}$ were screened for antibacterial activity by using the Kirby Bauer disc diffusion test on Mueller-Hinton agar plates. The medium was poured into Petri plates and allowed to solidify. These plates were inoculated with a bacterial inoculum prepared in physiologically sterile water with an OD of about 0.08. Sterilized disks of $6 \mathrm{~mm}$ (Schleicher and Schule, Germany) were each impregnated with $20 \mu \mathrm{L}$ of different concentrations of the compounds and were deposited on the plates. The latter were then left at room temperature for $2 \mathrm{~h}$ and incubated at $37^{\circ} \mathrm{C}$ for $24 \mathrm{~h}$. The diameters of the inhibition zones $(\mathrm{mm})$ were measured in accordance with the recommendations of the clinical and laboratory standards institute (CLSI 2017) [32]. For each bacterial strain, the best inhibition zone obtained was reported in Table 4.

Secondly, the MIC values were determined by the dilution broth method following the procedure recommended by the CLSI [32]. The serial dilutions of compounds, ranging from concentrations of 0.5 to $512 \mu \mathrm{g} / \mathrm{mL}$, T were inoculated with fresh bacterial inoculums and then incubated at $37^{\circ} \mathrm{C}$ for $24 \mathrm{~h}$. The MIC value was considered as the lowest concentration showing visual inhibition of 
growth. Sulfamethoxazole-trimethoprime (Bio-Rad, Marseille, France) was used as the positive control $(\mathrm{CMI}=25 \mu \mathrm{g} / \mathrm{mL})$. Disks embedded with acetone were used as a negative one.

\section{Conclusions}

In summary, 12 new and original sulfamidocarbonyloxyphosphonates were synthesized and fully characterized by ${ }^{1} \mathrm{H},{ }^{13} \mathrm{C}$, and ${ }^{31} \mathrm{P}$ NMR spectroscopy, IR spectroscopy, and mass spectroscopy, as well as elemental analysis. The synthesized compounds $\mathbf{1 a}-\mathbf{7} \mathbf{a}$ and $\mathbf{1} \mathbf{b}$ were screened for in vitro evaluation as a proof of concept for designing new antibacterial agents containing both sulfamido and phosphonate moieties. Standard strains were chosen according to the screening protocol including Gram-positive and Gram-negative bacteria, which represent micro-organisms associated with important infections. All compounds showed promising in vitro antibacterial activity. Additionally, it has been demonstrated that our derivatives have more antibacterial effects on Gram-negative bacteria than Gram-positive ones except for compound $\mathbf{1 b}\left(\mathrm{R}_{1}=\mathrm{CH}_{3}, \mathrm{R}_{2}=\mathrm{CH}(i \mathrm{Bu}) \mathrm{COOEt}, \mathrm{R}_{3}=\mathrm{H}\right)$. This latter is the only one active on both Gram-negative and Gram-positive bacteria. Compound $1 a\left(R_{1}=C_{3}, R_{2}=B n, R_{3}=H\right)$ had more pronounced activity against $P$. aeruginosa, whereas compound $4 \mathbf{a}\left(\mathrm{R}_{1}=\mathrm{CH}_{3}, \mathrm{R}_{2}=3-\mathrm{F}-\mathrm{C}_{6} \mathrm{H}_{4}, \mathrm{R}_{3}=\mathrm{H}\right)$ had more activity on E. coli. Antibacterial effects will be investigated in further studies to explain the susceptibility of bacteria to our compounds. Further pharmacomodulation efforts are in progress to explore the impact of new substituents on the phenyl moiety and thereby will offer new expectations for sulfamidocarbonyloxyphosphonates as novel antibacterial agents.

Supplementary Materials: Supplementary materials are available on line.

Author Contributions: A.B., K.B., and B.B. synthesized all compounds presented in this article; J.L. and C.M. contributed to the identification of all synthesized products by NMR and MS; I.B. and H.B. performed the bioassays of compounds; Z.B., C.M., and M.L.B. wrote and revised the paper; J.L. revised the paper; M.B. started the project, designed the molecules, and wrote and revised the paper.

Funding: APC was sponsored by MDPI. This research received no external funding.

Acknowledgments: This work was financially supported by The General Directorate for Scientific Research and Technological Development (DG-RSDT), Algerian Ministry of Scientific Research, Applied Organic Chemistry Laboratory (FNR 2000).

Conflicts of Interest: The authors declare no conflict of interest.

\section{References}

1. Spillane, W.; Malaubier, J.-B. Sulfamic acid and its $N$ - and O-substituted derivatives. Chem. Rev. 2014, 114, 2507-2586. [CrossRef] [PubMed]

2. Winum, J.Y.; Scozzafava, A.; Montero, J.L.; Supuran, C.T. Therapeutic potential of sulfamides as enzyme inhibitors. Med. Res. Rev. 2006, 26, 767-792. [CrossRef] [PubMed]

3. Reitz, A.B.; Smith, G.R.; Parker, M.H. The role of sulfamide derivatives in medicinal chemistry: A patent review (2006-2008). Expert Opin. Ther. Pat. 2009, 19, 1449-1453. [CrossRef] [PubMed]

4. Berredjem, H.; Reggami, Y.; Benlaifa, M.; Berredjem, M.; Bouzerna, N. Antidiabetic and hypolipidemic potential of 3,4-dihydroisoquinolin-2(1H)-sulfonamide in alloxan induced diabetic rats. Int. J. Pharm. 2015, 11, 226-235.

5. Suthagar, K.; Fairbanks, A.J. Synthesis and anti-mycobacterial activity of glycosyl sulfamides of arabinofuranose. Org. Biomol. Chem. 2016, 14, 1748-1754. [CrossRef] [PubMed]

6. Villalba, M.L.; Enrique, A.V.; Higgs, J.; Castaño, R.A.; Goicoechea, S.; Taborda, F.D.; Gavernet, L.; Lick, I.D.; Marder, M.; Bruno Blanch, L.E. Novel sulfamides and sulfamates derived from amino esters: Synthetic studies and anticonvulsant activity. Eur. J. Pharmacol. 2016, 774, 55-63. [CrossRef] [PubMed]

7. Becheker, I.; Berredjem, H.; Boufas, W.; Berredjem, M. The antibacterial and cytotoxic activities of four new sulfonamides against clinical Gram-negative bacteria. Int. J. Pharm. Sci. Rev. Res. 2016, 39, 125-133.

8. Berrino, E.; Bua, S.; Mori, M.; Botta, M.; Murthy, V.S.; Vijayakumar, V.; Tamboli, Y.; Bartolucci, G.; Mugelli, A.; Cerbai, E.; et al. Novel sulfamide-containing compounds as selective carbonic anhydrase I inhibitors. Molecules 2017, 22, 1049. [CrossRef] [PubMed] 
9. Juna, J.H.; Kumarb, V.; Dexheimerc, T.S.; Wedlichd, I.; Nicklausd, M.C.; Pommierc, Y.; Malhotra, S.V. Synthesis, anti-cancer screening and tyrosyl-DNA phosphodiesterase 1 (Tdp1) inhibition activity of novel piperidinyl sulfamides. Eur. J. Pharm. Sci. 2018, 111, 337-348. [CrossRef] [PubMed]

10. Anderson, K.K.; Barton, D.H.; Ollis, R.; Jones, W.D. Sulfonic Acids and Their Derivatives in Comprehensive Organic Chemistry; Pergamon Press: Oxford, UK, 1979; Volume 3, pp. 331-340.

11. Cano, C.; Paez, J.A.; Goya, P.; Serrano, A.; Pavon, J.; Rodriguez de Fonseca, F.; Suardiaz, M.; Martin, M.I. Synthesis and pharmacological evaluation of sulfamide-based analogues of anandamide. Eur. J. Med. Chem. 2009, 44, 4889-4895. [CrossRef] [PubMed]

12. Supuran, C.T.; Casini, A.; Mastrolorenzo, A.; Scozzafava, A. COX-2 selective inhibitors, carbonic anhydrase inhibition and anticancer properties of sulfonamides belonging to this class of pharmacological agents. Mini Rev. Med. Chem. 2004, 4, 625-632. [CrossRef] [PubMed]

13. Abbate, F.; Casini, A.; Owa, T.; Scozzafava, A.; Supuran, C.T. Carbonic anhydrase inhibitors: E7070, a sulfonamide anticancer agent, potently inhibits cytosolic isozymes I and II, and transmembrane, tumor-associated isozyme IX. Bioorg. Med. Chem. Lett. 2004, 14, 217-223. [CrossRef] [PubMed]

14. Swain, N.A.; Batchelor, D.; Beaudoin, S.; Bechle, B.M.; Bradley, P.A.; Brown, A.D.; Brown, B.; Butcher, K.J.; Butt, R.P.; Chapman, M.L.; et al. Discovery of clinical candidate 4-[2-(5-amino-1H-pyrazol-4yl)-4-chlorophenoxy]-5-chloro-2-fluoro-N-1,3-thiazol-4-ylbenzenesulfonamide (PF-05089771): Design and optimization of diaryl ether aryl sulfonamides as selective inhibitors of NaV1.7. J. Med. Chem. 2017, 60, 7029-7042. [CrossRef] [PubMed]

15. Abdoli, M.; Angeli, A.; Bozdag, M.; Carta, F.; Kakanejadifard, A.; Saeidian, H.; Supuran, C.T. Synthesis and carbonic anhydrase I, II, VII, and IX inhibition studies with a series of benzo[d]thiazole-5- and 6-sulfonamides. J. Enzyme Inhib. Med. Chem. 2017, 32, 1071-1078. [CrossRef] [PubMed]

16. Mojica, S.A.; Salin, O.; Bastidas, R.J.; Sunduru, N.; Hedenström, M.; Andersson, C.D.; Otero, C.N.; Engström, P.; Valdivia, R.H.; Elofsson, M.; Gylfe, A. N-acylated derivatives of sulfamethoxazole block Chlamydia fatty acid synthesis and interact with FabF. Antimicrob. Agents Chemother. 2017, 61, e00716-17. [CrossRef] [PubMed]

17. Biasone, A.; Tortorella, P.; Campestre, C.; Agamennone, M.; Preziuso, S.; Chiappini, M.; Nuti, E.; Carelli, P.; Rossello, A.; Mazza, F.; et al. $\alpha$-Biphenylsulfonylamino 2-methylpropyl phosphonates: Enantioselective synthesis and selective inhibition of MMPs. Bioorg. Med. Chem. 2007, 15, 791-799. [CrossRef] [PubMed]

18. Kim, D.K.; Young, J.; Lee, H.; Park, J.; Minh Thai, K. Synthesis and phosphodiesterase 5 inhibitory activity of new sildenafil analogues containing a phosphonate group in the $5^{\prime}$-sulfonamide moiety of phenyl ring. Bioorg. Med. Chem. Lett. 2004, 14, 2099-2103. [CrossRef] [PubMed]

19. Holmes, C.P.; Li, X.; Pan, Y.; Xu, C.; Bhandari, A.; Moody, C.M.; Miguel, J.A.; Ferla, S.W.; De Francisco, M.N.; Frederick, B.T.; et al. Discovery and structure-activity relationships of novel sulfonamides as potent PTP1B inhibitors. Bioorg. Med. Chem. Lett. 2005, 15, 4336-4341. [CrossRef] [PubMed]

20. Watkins, W.J.; Cho, A. Preparation of Phosphonate Derivatives of Mycophenolic Acid as Anticancer, Antiviral and Anti-Inflammatory Agents. WO2006047661, 4 May 2006.

21. Timmler, H.; Wegler, R.; Unterstenhafer, G. Procédé de Fabrication de Dérivés Phosphorylés de Sulfonyle Urethanes. FR1,403,523A, 18 June 1965.

22. Winum, J.Y.; Bouissiere, J.L.; Passagne, I.; Evrard, A.; Montero, V.; Cuq, P.; Montero, J.L. Synthesis and biological evaluation of fotemustine analogues on human melanoma cell lines. Eur. J. Med. Chem. 2003, 38, 319-324. [CrossRef]

23. Bartzatt, R.; Cirillo, S.L.; Cirillo, J.D. Sulfonamide agents for treatment of Staphylococcus MRSA and MSSA infections of the central nervous system. Cent. Nerv. Syst. Agents Med. Chem. 2010, 10, 84-90. [CrossRef] [PubMed]

24. Sköld, O. Sulfonamide resistance: Mechanisms and trends. Drug Resist. Updates 2000, 3, 155-160. [CrossRef] [PubMed]

25. Huovinen, P. Resistance to trimethoprim-sulfamethoxazole. Clin. Infect. Dis. 2001, 32, 1608-1614. [PubMed]

26. Karageorgopoulos, D.E.; Wang, R.; Yu, X.H.; Falagas, M.E. Fosfomycin: Evaluation of the published evidence on the emergence of antimicrobial resistance in Gram-negative pathogens. J. Antimicrob. Chemother. 2012, 67, 255-268. [CrossRef] [PubMed]

27. Rossolini, G.M.; Mantengoli, E. Antimicrobial resistance in Europe and its potential impact on empirical therapy. Clin. Microbiol. Infect. 2008, 14 (Suppl. 6), 2-8. [CrossRef] [PubMed] 
28. Cheloufi, H.; Berredjem, M.; Boufas, W.; Bouchareb, F.; Aouf, N.E. Efficient synthesis, characterization, and antibacterial activity of novel $\mathrm{N}$-acylsulfonamides and sulfonylureas. Phosphorus Sulfur Silicon Relat. Elem. 2014, 189, 1396-1404.

29. Boufas, W.; Cheloufi, H.; Bouchareb, F.; Berredjem, M.; Aouf, N.E. Convenient synthesis of novel N-acylsulfonamides containing phosphonates moiety. Phosphorus Sulfur Silicon Relat. Elem. 2015, 190, 103-111. [CrossRef]

30. Bouchareb, F.; Berredjem, M.; Ait Kaki, S.; Bouaricha, A.; Bouzina, A.; Belhani, B.; Aouf, N.E. Synthesis and antibacterial activity of new chiral $N$-sulfamoyloxazolidin-2-ones. J. Chem. Sci. 2016, 128, 85-91. [CrossRef]

31. Bouzina, A.; Grib, I.; Bechlem, K.; Belhani, B.; Aouf, N.E.; Berredjem, M. Efficient synthesis of novel $N$-acylsulfonamide oxazolidin-2-ones derivatives. Karbala Int. J. Mod. Sci. 2016, 2, 98-103. [CrossRef]

32. Clinical and Laboratory Standards Institute (CLSI). Performance Standards for Antimicrobial Susceptibility Testing, 27th ed.; Clinical and Laboratory Standards Institute Antimicrobial Susceptibility Testing Standards M02-M07; CLSI: Wayne, PA, USA, 2017.

33. Sobhani, S.; Tashrifi, Z. Synthesis of $\alpha$-functionalized phosphonates from $\alpha$-hydroxyphosphonates. Tetrahedron 2010, 66, 1429-1439. [CrossRef]

34. Bouzina, A.; Aouf, N.E.; Berredjem, M. Ultrasound assisted green synthesis of $\alpha$-hydroxyphosphonates under solvent free conditions. Res. Chem. Intermed. 2016, 42, 5993-6002. [CrossRef]

35. Kaboudin, B.; Afsharinezhad, M.B.; Yokomatsu, T. A convenient and general procedure for the synthesis of $\alpha$-ureidophosphonates under catalyst-free conditions. Arkivoc 2012, 4, 44-53.

36. Hu, G.; Chen, W.; Ma, D.; Zhang, Y.; Xu, P.; Gao, Y.; Zhao, Y. Silver-catalyzed, aldehyde-induced $\alpha-\mathrm{C}-\mathrm{H}$ functionalization of tetrahydroisoquinolines with concurrent $\mathrm{C}-\mathrm{P}$ bond formation/ $\mathrm{N}$-alkylation. J. Org. Chem. 2016, 81, 1704-1711. [CrossRef] [PubMed]

Sample Availability: Samples of the synthesized compounds are available from the corresponding authors.

(C) 2018 by the authors. Licensee MDPI, Basel, Switzerland. This article is an open access article distributed under the terms and conditions of the Creative Commons Attribution (CC BY) license (http://creativecommons.org/licenses/by/4.0/). 